\title{
Use of Telephone Calls in Reduction of Dropout Rate of Routine Immunization in a Tertiary Health Facility in South Eastern Nigeria
}

\author{
Nwokeukwu Huldah Ijeoma ${ }^{1}$, Emma-Ukaegbu Uloaku', Ajuogu Eno ${ }^{1}$, Osunkwo Damaris ${ }^{2}$, \\ Asinobi Adanze ${ }^{3}$ \\ ${ }^{1}$ Department of Community Medicine, Federal Medical Centre, Umuahia, Abia State, Nigeria \\ ${ }^{2}$ Department of Internal Medicine, National Hospital Abuja, Federal Capital Territory, Abuja, Nigeria \\ ${ }^{3}$ Department of Pediatrics, University College Hospital, Ibadan, Oyo State, Nigeria
}

Email address:

drhiln@yahoo.com (N. H. Ijeoma)

\section{To cite this article:}

Nwokeukwu Huldah Ijeoma, Emma-Ukaegbu Uloaku, Ajuogu Eno, Osunkwo Damaris, Asinobi Adanze. Use of Telephone Calls in Reduction of Dropout Rate of Routine Immunization in a Tertiary Health Facility in South Eastern Nigeria. European Journal of Preventive Medicine. Vol. 3, No. 3, 2015, pp. 39-43. doi: 10.11648/j.ejpm.20150303.11

\begin{abstract}
Background: In Federal Medical Centre (FMC) Umuahia, DPT dropout rate has been high above 10\% (2009 18\% and 2010 16\% source immunization unit Federal Medical centre, Umuahia) which indicates poor utilization of the Health Facility. It was therefore necessary to use recall telephone system to see if it could be reduced. Objectives: To determine the use of recall on and causes of immunization dropout. Methodology: This was an interventional study, in which all the children immunized in April 2011 who were given BCG to start the immunization regimen, were followed up until they completed the immunization with measles at nine months. Prior to this, dropout rate was calculated for three preceding months using the immunization register. The reason for dropout was obtained from the caregivers. The dropout rate was also calculated for the month of May. Result: The dropout rate reduced when compared with the previous months. Total number of children immunized with BCG in April 2011 was 119 and those who received DPT1 107, DPT2 96, DPT3 92 and measles 73. The cumulative DPT dropout rate in April was $14 \%$ while it was $20.8 \%$ in March. The most important cause of dropout was distance. Conclusion/ Recommendation: The recall improved the dropout rate, though not less than 10\%. This showed that if the caregivers are recalled some will come back for immunization. The cause of the dropout was mainly distance. FMC Umuahia being a tertiary health facility with experts, many women put to birth in that hospital and their babies will be given BCG at birth and DPTI when they come for 6 weeks post natal and then dropout. Recall system should be instituted in all the health facilities to improve utilization. A column should be provided in the child immunization register for writing the caregivers phone number.
\end{abstract}

Keywords: Drop-Out, Telephone Calls, Immunization

\section{Introduction}

Vaccine preventable diseases remain the most common causes of childhood mortality with an estimated three million deaths each year ${ }^{1}$. Immunization reminder/recall systems are cost-effective methods whereby children in need of vaccination are identified and contacted to come to the physician's office. Reminder systems track future appointments, whereas recall systems track missed appointments during which immunizations would have been given $^{2}$. Different methods exist such as chart reminders, mail reminders, postcards, telephone calls, home visits, computerized immunization alert systems, standing orders, expanding clinic hours, card file, Clinical Assessment Software Application (CASA). However no one method can be said to suit all facilities. The various methods are dependent upon office personnel's ability to utilize the selected method and fine-tune it to meet the specific needs of the practice and locale.

Immunization dropout and immunization coverage rates at Primary Health Care (PHC) level indicate the level at which communities utilize the preventive services and thus serve as a proxy of the strength of the public health system. They also 
measure the effectiveness of the immunization programme ${ }^{3}$. Dropouts in immunization refer to children who have used immunization services, but do not return for subsequent vaccinations and should not exceed 10 percent; beyond that, it indicates a problem of either access or utilization of services. In most settings where full immunization coverage is low, most children receive at least one dose of DPT, but the proportion that receive the needed second and third doses drops steeply. Dropout rates are calculated as the percentage point difference between successive doses of a vaccine, expressed as a percentage of the first dose. For example, the dropout rate between the first(DPT1) and third (DPT3) doses of DPT is calculated by subtracting the cumulative total for DTP3 from the cumulative total for DTP1 divided by the cumulative of DPT1 all multiplied by $100 \%$. (DPT1DPT3)/DPT $1^{3}$.

The second indicator of utilization of the preventive services at PHC level, which is immunization coverage, is measured by the percentage of children under one year old who have received the appropriate immunizations. The indicator is calculated as the total number of children under one year old that have received all their immunizations up to the first measles, divided by the total population of children under one year old in a given area. This study was carried out to institute and determine the effect of the use of mobile phone recall on immunization dropout rate in FMC Umuahia.

\section{Materials and Methods}

\subsection{Study Area}

The study was carried out in the National Programme of Immunization unit in FMC Umuahia, the only tertiary health institution in Umuahia, the capital of Abia State, Nigeria. The institution has a Community Medicine department which runs the immunization unit since its inception in 1999. The clinic delivers vaccines to both children and adults. It is open and operational on a daily basis, but due to logistics reasons, BCG, Measles and Yellow fever vaccines are given only once a week whereas the other antigens once available are given as clients come in. The catchment area by the facility includes Okpara Avenue, Library Avenue, FMC Umuahia, Factory Road, Railway Quarters, Umuobasi and Isiama Afara. It is bounded by Isiama Afara in the south, Ugba in the north World Bank in the East and Mbaocha in the West. The total population is 30,600 and an annual target population (011 months) of 1224 .

\subsection{Study Design}

This was an interventional study. Commencing from the beginning of the month of April 2011, a comprehensive record of phone numbers of all the clients who brought their children to receive BCG injections was collected alongside other relevant data in the child immunization register. Calls (Recalls) were made to those who missed their appointed dates for immunization after four weeks. All the 119 clients that commenced their childhood immunizations in the month of April participated in the study. Prior to this the drop-out rate from January to March the same year was calculated. Also the drop-out rate for the month of may without recall was also calculated.

In order to categorize a child as a "dropout ", a minimum of 4weeks would have elapsed because this is the interval between the doses for DPT. Once a client missed an appointment, at least three phone calls were made to ascertain the reason and also to urge the patient to come on the date agreed upon by the health worker and the patient. All the new agreed dates were written and rechecked to ensure that the patient actually came to receive the vaccine.

\subsection{Data Collection and Analysis}

The baseline data was obtained from the first quarter data which was obtained from the immunization register and following the institution of the intervention in the month of April. The clients were followed up till all the antigens were received. The variables derived from the child immunization register and the phone conversations were entered and analyzed using Excel and EPI-INFO version 3.4

\subsection{Ethical Consideration}

The ethical clearance was given by the Ethical committee of FMC, Umuahia, Abia State Nigeria with reference number FMC/QEH/G.596/4/63.

\section{Results}

Annual Target population of $0-11$ months was 1224 . The total number that registered for the immunization in the month of April 2011 was 119; Males 63 (53\%), females $56(47 \%)$. The total recalls made were 80 but at different levels. The number that responded positively was $56(70 \%)$. All the clients that did not come back received immunization in another Health Facility (HF). The dropout rate was virtually the same for both male (51\%) and female (49\%). As shown in figure $\mathrm{I}$, there was a reduction in BCG/Measles dropout from $45 \%$ for the month of March to $37 \%$ for the month of April showing a decline of $8 \%$. There was also a rise in the month of May again to $47 \%$ showing a $10 \%$ increase. Similarly, the dropout between DPT1 and DPT3 fell from $21 \%$ to $14 \%$, showing a downward trend of $7 \%$. There was a rise again in the month of May to $22 \%$ showing an increase of $8 \%$; $\mathrm{P}$ value 0.25 and 0.2 respectively. These figures are not statistically significant. Importantly, dropout between the various vaccines remained constant also except between DPT2 and DPT3 in the month of April when it fell from $20 \%$ in March to $10 \%$ in April, $\mathrm{P}$ value 0.05 ; as shown in figure II.

The reasons gathered from caretakers of the children showed that most of them $49 \%$ could not come back because of distance (see figure III). Some came to deliver in the hospital and after went back to their residence where they continued the immunization. Only one (2\%) woman indicated the death of the child. 


\section{Discussion}

This study showed improvements with the inception of the recall intervention and this is similar to studies done in a Pennsylvanian pilot study, using phone recall, the health staff were successful in locating and bringing in the overdue children and this resulted in approximately half $(50 \%)$ of the overdue children being brought up-to-date for immunization ${ }^{5}$.

Compared with other methods of recall, a study found that telephone reminders resulted in $37 \%$ of persons receiving influenza vaccine compared with $9.8 \%$ in a randomized control group ${ }^{6}$. Studies on telephone reminders have shown positive results in improving the rate of appointments kept. ${ }^{7-12}$

In a Cochrane review by Szilagyi et al they found that patient reminder/recall systems were effective in improving immunization rates in 33 of 41 studies. Increases in immunization rates due to reminders were in the range of 5 to 20 percentage points ${ }^{13}$. In another study by Ahlers-Schmidt et al to test the effect of text reminders on immunization compliance in kids, they found that a greater number of children in the intervention group that received text message reminders turned up more for immunization as against the control who received none. The result though was not statistically significant ${ }^{14}$. Similarly, a study in Addis Ababa showed that the use of reminders in the form of stickers reduced dropout rate amongst children ${ }^{15}$.

On the contrary, a study carried out by Daley et al failed to demonstrate any significant increase in the study population comprising of an inner city disadvantaged group in the US ${ }^{16}$.

In this study, care givers did not demonstrate a lack of commitment to immunization, rather they gave distance in figure III as a reason why they could not come back to FMC for further vaccines, and they actually continued the immunization in centers close to their residential area. In a study by Itimi et al mothers were not returning for immunization due to lack of motivation, poor attitude of the health workers and relocation to a new environment ${ }^{17}$. Phukan et al demonstrated lack of information on immunization on the part of the parents as the reason for dropout ${ }^{18}$. However the reason in this study could be that, the first doses which were received in the study center coincided with the mother's post natal care check up visits. When there was no longer a need for the mothers to return to the center for their own check-up, they now went to nearer centers for their children's immunization. The fact that immunization was continued in nearby health centers was an indication that these group of parents actually are aware of the importance of this intervention. A recall system may be more effective in studies where attitude is the incriminating factor for dropping out of immunization. The recall would then act as a motivator for those concerned. It is also interesting to note that the study demonstrated equal dropout in both males and females with that of the males being marginally higher though not statistically significant.

Although the minimal reduction in the dropout rate between March and April was not statistically significant, the magnitude of its public health importance cannot be overemphasized. For every child recalled for immunization, so much has been done to prevent disease, morbidity and mortality in that child, prevent disabilities as well as disease transmission to many others who might have gotten infected through the child and also boosting herd immunity.

\section{Limitation of Study}

The scarcity of vaccine in the year which reduced the period of study. Unavailability of telephone numbers of few care givers.

\section{Conclusion}

Although the dropout rate reduced, it did not get below $10 \%$ which is the target of good utilization. The combination of recall and reminder may give a better result. Provision should be made in the immunization register to include the phone numbers of the caregivers. Measles should be given at least twice a week to reduce the missed opportunity in the health facility. Further study can also be done in a rural health facility to compare the results.

\section{Acknowledgement}

The effort of the nursing staff in writing the phone numbers of the care givers in the child immunization register is acknowledged.

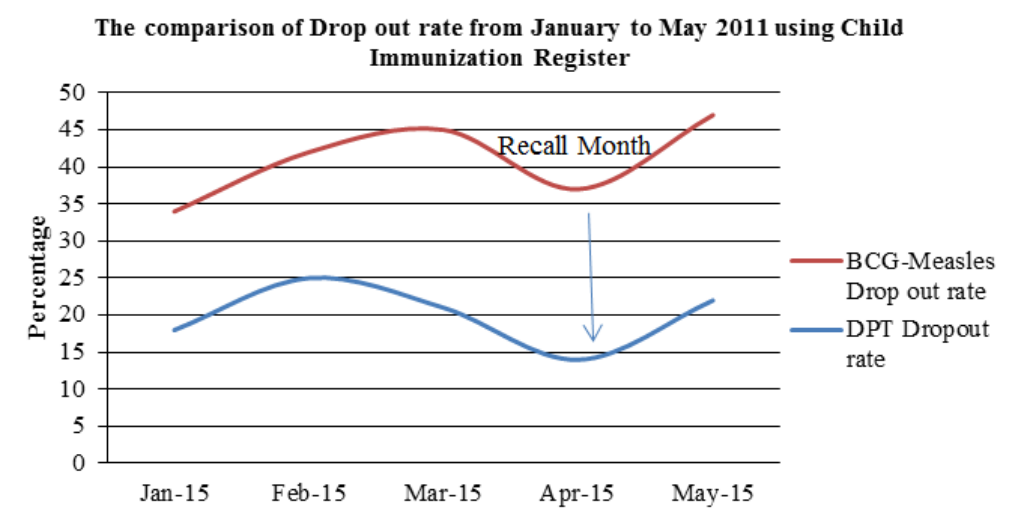

Figure I. Comparison of dropout rate from January to may 2011. 
The rate of Drop from one vaccine to the other Jan-May 2011

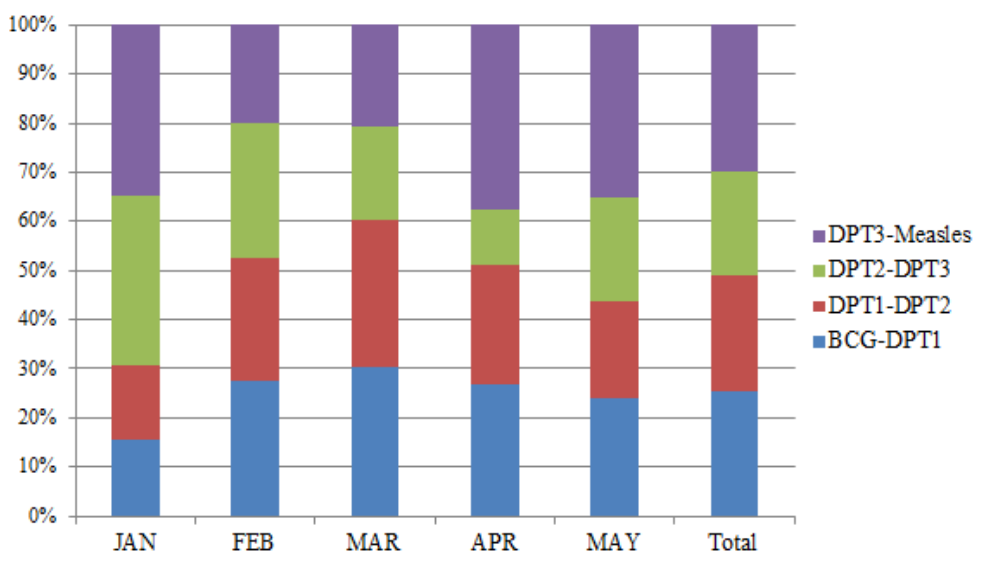

Figure II. The monthly rate of drop from one antigen to the other.

\section{Causes of Drop out rate in the recalled Month April 2011}

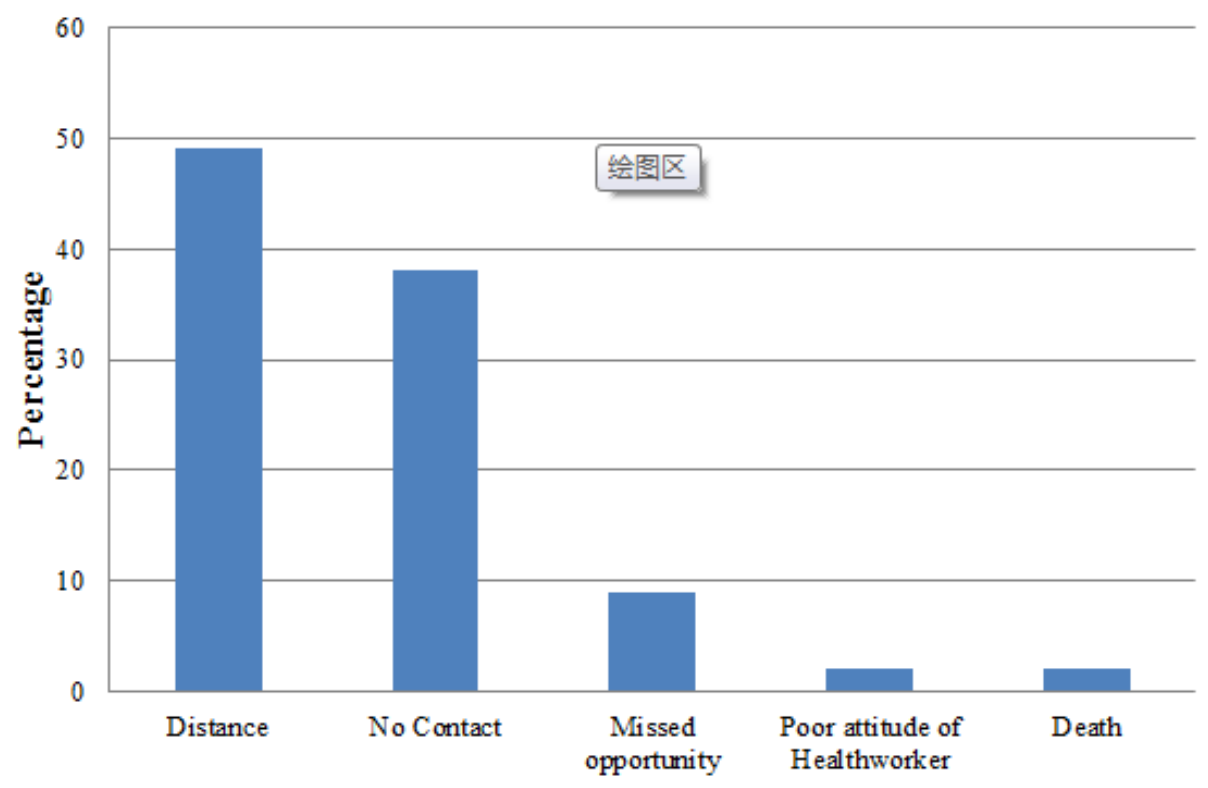

Figure III. Causes of dropout.

\section{References}

[1] Centre for Global Development: Making Markets for vaccines: from ideas to actions. Centre for Global Development; Washington DC; 2005.

[2] Vaccine Reminder Recall Systems: A Practical Guide for Pediatric Practices http://practice.aap.org/content.aspx?aid $=2674$ accessed on $7^{\text {th }}$ of April 2011

[3] Immunization Dropout- HMIS News. Htm. Sept 222011 pg 1

[4] S.S. Trivedi, C.R. Mundada \& R.K. Chudasama: Evaluation and impact of various factors affecting Universal Immunization Programme (UIP) coverage in Surat. The Internet Journal of Epidemiology. 2009; 6:2

[5] Kathleen Marker, RN. Successful Implementation of Practicebased Reminder/Recall Systems. Implementation of a Vaccine
Reminder/Recall System: A Successful Pilot Project in Pennsylvania

[6] Mc Dowell, Newell C., Rosser W. Comparism of methods of recalling influenza vaccination. Canadian Medical Journal 1986; 135:991-997.

[7] Muhammad J H, Peter F, and Kevin F. Effectiveness of Telephone Reminders in Improving Rate of Appointments Kept at an Outpatient Clinic: A Randomized Controlled Trial .J Am Board Fam Med. 2001;14(3)

[8] Reekie D, Devlin H. Preventing failed appointments in general dental practice: a comparison of reminder methods. $\mathrm{Br}$ Dent J 1998; 185:472- 4.

[9] O'Brien G, Lazebnik R. Telephone call reminders and attendance in an adolescent clinic. Pediatrics 1998; 101:E6.

[10] Koren ME, Bartel JC, Corliss J. Interventions to improve patient appointments in an ambulatory care facility. J Ambulatory Care Manage 1994;17:76 
[11] Grover S, Gagnon G, Flegel KM, Hoey JR. Improving appointment-keeping by patients new to a hospital medical clinic with telephone or mailed reminders. Can Med Assoc J 1983;129:1101-3

[12] Cohen AJ, Weinstein P, Wurster C. The effects of patientinitiated phone confirmation strategies on appointment keeping at a hospital dental clinic. J Public Health Dent 1980;40:64 -8.

[13] Szilagyi P, Vann J, et al. interventions aimed at improving immunization

rates. http://www.ncbi.nlm.nih.gov/pubmed/12519624. Assessed $30 / 1 / 13$.

[14] Ahlers-Schmidts CR, Chesser AK, NguyenT. Feasibility of a Randomized Controlled Trial to evaluate Text Reminders for immunization Compliance in Kids. (TRICKs) http://www.ncbi.nlm.nih.gov/pubmed/22750044. Assessed on $4 / 2 / 2013$.
[15] Berhane Y, Pickering J. Are reminder stickers effective in reducing immunization dropout rates in Addis Ababa, Ethiopia? http://www.ncbi.nlm.nih.gov/pubmed/8505766

[16] Daley MF, Steiner JF, Brayden RM, et al. Immunization registry-based recall for a new vaccine. Ambul Pediatr. 2002;2:438-443. CrossRefMedlineWeb of Science

[17] Kalamawei Itimi, Paul O Dienye, Best Ordinioha. Community participation and childhood immunization coverage: A comparative study of rural and urban communities of Bayelsa State, south-south Nigeria. Nigerian Medical Journal, Year 2012, Volume 53, Issue 1 [p. 21-25]

[18] Phukan RK, Barman MP, Mahanta J. factors associated with immunization coverage of children in Assam, India: over the first year of life. http://www.ncbi.nlm.nih.gov/pubmed/18450821. Assessed on 4/2/2013. 